\title{
Clinical Implications of the New Equation to Estimate Glomerular Filtration Rate
}

\author{
Davide Giavarina $^{a}$ Faeq Husain-Syed ${ }^{b, c}$ Claudio Ronco ${ }^{c, d}$ \\ ${ }^{a}$ Department of Clinical Chemistry and Hematology Laboratory, San Bortolo Hospital, Vicenza, Italy; ${ }^{b}$ Department of \\ Internal Medicine II, University Hospital Giessen, Justus-Liebig-University Giessen, Giessen, Germany; 'Department \\ of Nephrology, Dialysis and Transplantation, International Renal Research Institute of Vicenza, San Bortolo Hospital,

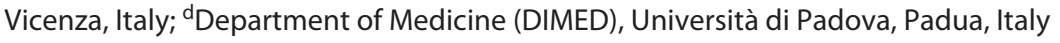

\section{Keywords}

Chronic kidney disease - Chronic kidney disease epidemiology collaboration · European kidney function consortium - Glomerular filtration rate - Modification of diet in renal disease

\begin{abstract}
Recently, a new full-age spectrum equation was proposed by the European Kidney Function Consortium (EKFC) to overcome the difficulty of using multiple glomerular filtration rate (GFR) estimation equations and problems of implausible changes in GFR during the transition from adolescence to adulthood and address GFR overestimation in young adults and in the older adults. To verify the impact on patient classifications, we applied the new equation to data of 38,188 adult patients, comparing GFR estimation using the Chronic Kidney Disease Epidemiology Collaboration (CKD-EPI) and EKFC equations. As expected, our data indicate that a significant proportion of patients will be reclassified downward by the EKFC compared to the CKD-EPI equation with a particular reference between CKD stages 1-2 and $2-3$ and age categories of $18-30$ and $\geq 61$ years, respectively.
\end{abstract}

Clinicians should be aware that any replacement for the EKFC equation will entail a period of different results in estimated GFR during the transition from the previous to the new equation.

(c) 2021 S. Karger AG, Basel

Glomerular filtration rate (GFR) is widely accepted as the best overall index of kidney function and is an independent predictor of kidney failure and cardiovascular and all-cause mortality $[1,2]$. Thus, its assessment is essential for clinical practice, research, and public health. In 2010, we did a comparative study on GFR estimation by using the Modification of Diet in Renal Disease equation and (at that time) the new Chronic Kidney Disease Epidemiology Collaboration (CKD-EPI) equation [3]. The article was based on the analysis of $\sim 38,000$ serum creatinine ( $\mathrm{SCr}$ ) measurements and concluded that, in line with previous reports [4], the use of the CKD-EPI equation compared to the Modification of Diet in Renal Disease equation results in a lower estimated CKD prevalence. In a 2012 update to its CKD guideline, the Kidney Disease: Improving Global Outcomes-recommended estimating GFR using the CKD-EPI equation unless other karger@karger.com www.karger.com/nef
(C) 2021 S. Karger AG, Basel

Karger'
Correspondence to:

Claudio Ronco, claudio.ronco@unipd.it 


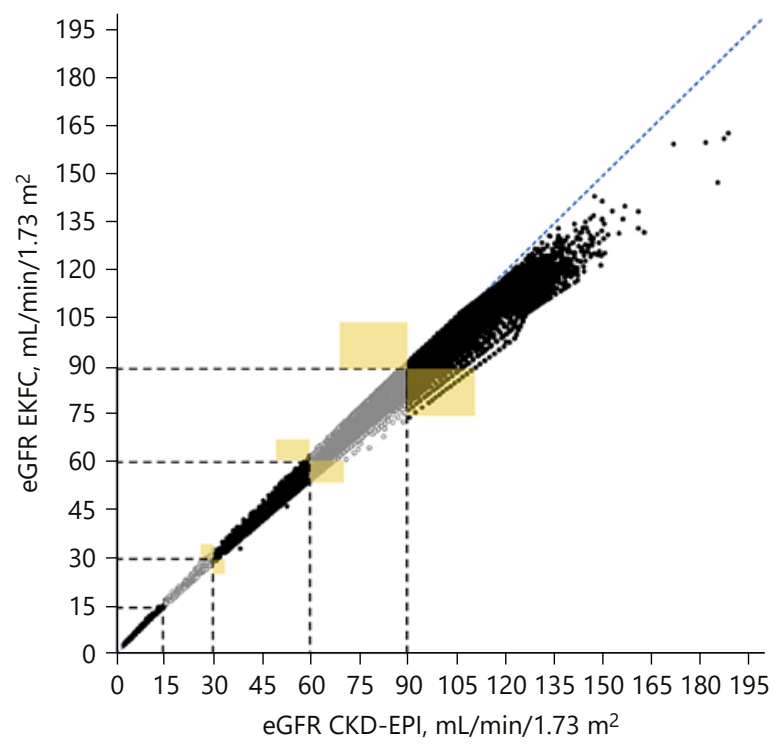

Fig. 1. Scatterplot of eGFR by CKD-EPI and EKFC equations the figure illustrates the overall correlation between eGFR values determined using the CKD-EPI ( $X$-axis) and EKFC ( $Y$-axis) equations based on SCr obtained from a previously described cohort [3], including 38,188 in-hospital and outpatients aged 18-75 years between May 1 and October 2009 at the central laboratory of San Bortolo Hospital, Vicenza, Italy. The dashed lines indicate the GFR cutoffs for CKD stages as defined by KDIGO [5]. The orange boxes delimit the cases of disagreement between both equations. CKD-EPI, Chronic Kidney Disease Equation Collaboration; eGFR, estimated glomerular filtration rate; EKFC, European Kidney Function Consortium; GFR, glomerular filtration rate; KDIGO, Kidney Disease: Global Improving Outcomes; SCr, serum creatinine.

equations are more accurate [5]. Since then, the CKD-EPI equation has increasingly consolidated in clinical and laboratory practice. Furthermore, in most clinical situations, it is used for the "first-line" test of kidney function [6]. However, several limitations remain, such as its applicability only in adults and the imprecision across different ethnic backgrounds [7].

Recently, to overcome the difficulty of using multiple equations and problems of implausible changes in GFR during the transition from adolescence to adulthood and address GFR overestimation in young adults and the older adults, a new full-age spectrum equation was proposed by the European Kidney Function Consortium (EKFC) [8]. The equation, based on data of 11,251 participants in 7 clinical studies, was validated using data of 8,378 participants in 6 other clinical studies. The validation showed improved accuracy and precision compared to those shown by commonly used equations for estimating GFR from SCr levels, particularly the Kidney Disease: Improving Global Outcomes-recommended CKD-EPI equation for adults and the Chronic Kidney Disease in Children Study equation for children [5].

To verify the possible impact of the equation change on our population, we applied the new equation to our data (real-world data), comparing GFR estimation using the CKD-EPI and EKFC equations, to verify the impact on our patient classifications. The details of the cohort have been described elsewhere [3].

Our data confirmed a good correlation between the 2 equations (shown in Fig. 1; further details are provided in the online suppl. data; for all online suppl. material, see www.karger.com/doi/10.1159/000516638). However, there was a significant bias in patient classification, with a particular reference between CKD stages 1-2 and 2-3 (shown in Table 1). We found that $19.6 \%$ of participants with an estimated GFR (eGFR) of $\geq 90$ $\mathrm{mL} / \mathrm{min} / 1.73 \mathrm{~m}^{2}$ (corresponding to stage $1 \mathrm{CKD}$ ) using the CKD-EPI equation had eGFR values of $60-90 \mathrm{~mL} /$ $\mathrm{min} / 1.73 \mathrm{~m}^{2}$ using the EKFC equation (corresponding to stage $2 \mathrm{CKD}$ ). Furthermore, $4.8 \%$ of participants with eGFR values of $60-89 \mathrm{~mL} / \mathrm{min} / 1.73 \mathrm{~m}^{2}$ using the CKD-EPI equation had eGFR values of $30-59 \mathrm{~mL} /$ $\mathrm{min} / 1.73 \mathrm{~m}^{2}$ (corresponding to stage $3 \mathrm{CKD}$ ) using the EKFC equation. Furthermore, when we analyzed eGFR by age categories, the EKFC equation led to lower eGFR values compared to the CKD-EPI equation, particularly in young adults (18-30 years) and in the older adults ( $\geq 65$ years) (shown in Table 2 ).

As expected, our data indicate that a significant proportion of patients will be reclassified downward by the EKFC compared to the CKD-EPI equation when SCr, age, and sex are randomly varied. We cannot evaluate the accuracy of both equations from the current study since measured GFR was not available; however, our data indicate that in line with Pottel et al. [8] the EKFC equation shows improved accuracy and precision compared with the CKD-EPI equation for estimating GFR, especially in young adults and the older adults. Consequently, clinicians should be aware that any replacement for the EKFC equation will entail a period of different results in eGFR during the transition from the previous to the new equation.

Our data refer to an unselected Italian population without any information related to ethnicity. Considering that $\sim 15 \%$ of the population in Northern Italy comes from Africa and Asia our results are likely to reflect the 


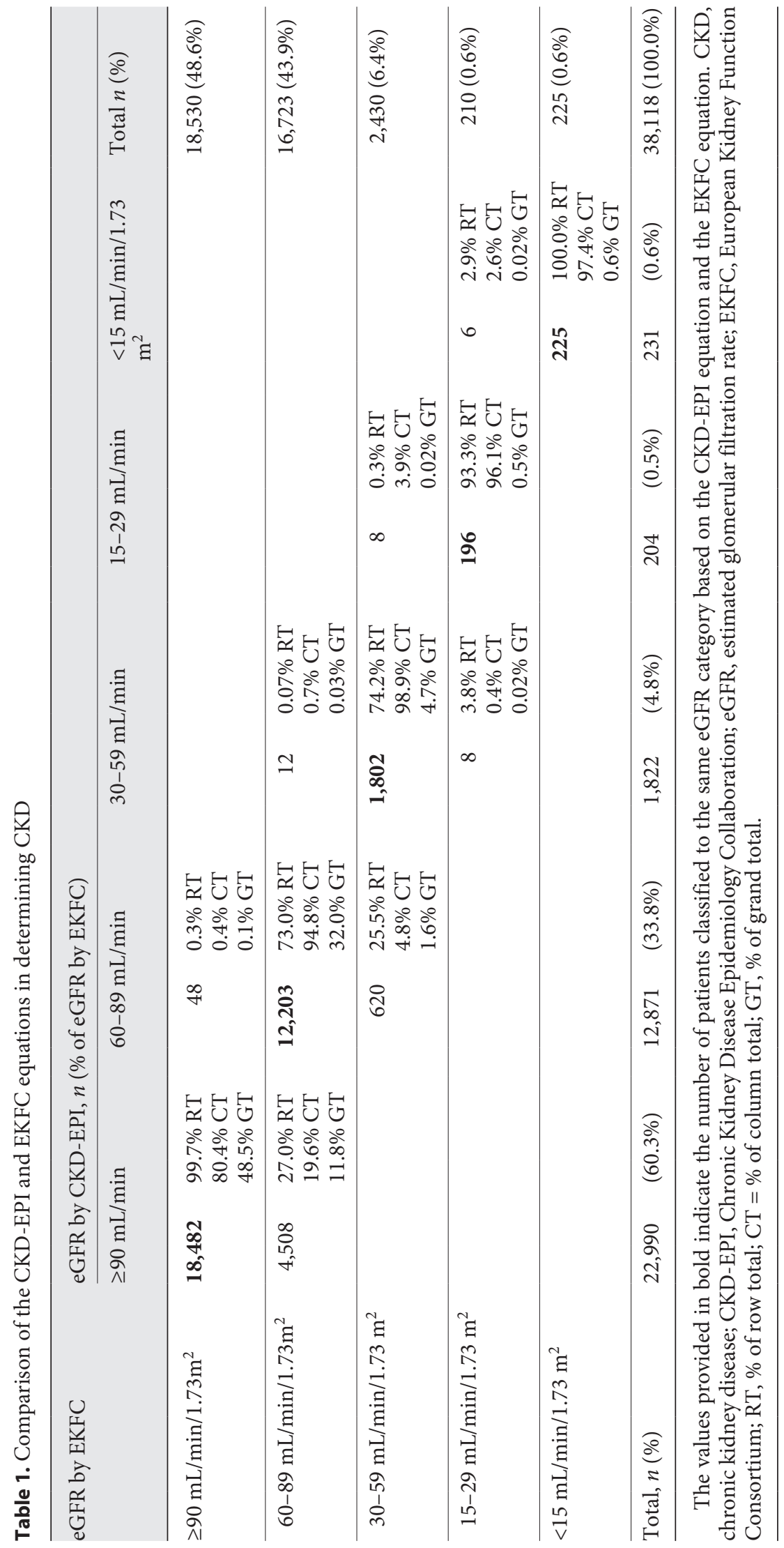


Table 2. Age-related distribution of eGFR using the CKD-EPI and EKFC equations

\begin{tabular}{lllll}
\hline Age, years & $N$ & $\begin{array}{l}\text { eGFR by CKD-EPI, } \\
\mathrm{mL} / \mathrm{min} / 1.73 \mathrm{~m}^{2}\end{array}$ & $\begin{array}{l}\text { eGFR by EKFC, } \\
\mathrm{mL} / \mathrm{min} / 1.73 \mathrm{~m}^{2}\end{array}$ & $p$ value \\
\hline $18-30$ & 4,606 & $118(105-125)$ & $106(95-111)$ & $<0.0001$ \\
$31-40$ & 6,345 & $109(97-115)$ & $106(106-107)$ & $<0.0001$ \\
$41-50$ & 7,635 & $101(89-107)$ & $99(88-105)$ & $<0.0001$ \\
$51-60$ & 7,422 & $93(81-100)$ & $89(78-95)$ & $<0.0001$ \\
$61-70$ & 8,683 & $84(71-92)$ & $78(67-85)$ & $<0.0001$ \\
$71-75$ & 3,427 & $76(63-87)$ & $70(58-79)$ & $<0.0001$ \\
\hline
\end{tabular}

Variables are expressed as median (interquartile range) values. eGFR, values were compared between both groups using the Wilcoxon signed-rank test. CKD-EPI, Chronic Kidney Disease Epidemiology Collaboration; eGFR, estimated glomerular filtration rate; EKFC, European Kidney Function Consortium. real-world application of the EKFC equation in Italy and Europe. Notably, the EKFC development population only included White European participants, which currently limits its generalization to more diverse ethnic populations. Recently, however, an interim statement of the $\mathrm{Na}$ tional Kidney Foundation and the American Society of Nephrology Task Force recommended that ethnicity modifiers should not be included in equations to estimate renal function [9]. Thus, the limitation of the EKFC equation in respect to ethnicity may not be considered as such in the foreseeable future.

In summary, the new EKFC equation is associated with various aspects of novelty and improvement. The conceptual advantage of using a single equation for all ages is obvious and could eliminate the discontinuity in eGFR during the transition from adolescence to adulthood and GFR overestimation in young adults. However, it may be important to promote an understanding of its strengths and limitations among clinicians since a significant proportion of patients might be reclassified downward by the EKFC compared to the CKD-EPI equation. Furthermore, a strong disclosing operation might be necessary to facilitate a rapid change and harmonization between laboratories in order to avoid worsening of the current estimates of GFR.

\section{Conflict of Interest Statement}

C.R. reports support for acting as an advisory board member for ASAHI, Baxter, GE, Jafron, and Medtronic, and speaker's fees from Astute, bioMérieux, B. Braun, Cytosorbents, ESTOR, FMC, and Toray, all unrelated to this work. D.G. and F.H.-S. declare no competing interests.

\section{Funding Sources}

This study did not receive external funding.

\section{Author Contributions}

All the authors contributed to the manuscript. D.G. drafted the manuscript and designed the figures and tables. F.H.-S. wrote part of the manuscript and reviewed several drafts. C.R. conceived the outline and wrote part of the manuscript and revised it in its final form in conjunction with all authors. C.R. is the senior author and attests that the authors had access to all the study data, and takes responsibility for the accuracy of the analysis, and had authority over manuscript preparation and the decision to submit the manuscript for publication.

\section{Availability of Data and Material}

The datasets used and/or analyzed during the study are available from the corresponding author upon reasonable request.

\section{References}

1 Chronic Kidney Disease Prognosis Consortium; Matsushita K, van der Velde M, Astor BC, Woodward M, Levey AS, et al. Association of estimated glomerular filtration rate and albuminuria with all-cause and cardiovascular mortality in general population cohorts: a collaborative meta-analysis. Lancet. 2010;375(9731):2073-81.

2 Gansevoort RT, Matsushita K, van der Velde M, Astor BC, Woodward M, Levey AS, et al. Lower estimated GFR and higher albuminuria are associated with adverse kidney outcomes. A collaborative meta-analysis of general and high-risk population cohorts. Kidney Int. 2011;80(1):93-104. 
3 Giavarina D, Cruz DN, Soffiati G, Ronco C. Comparison of estimated glomerular filtration rate (eGFR) using the MDRD and CKDEPI equations for CKD screening in a large population. Clin Nephrol. 2010;74(5):35863.

4 Earley A, Miskulin D, Lamb EJ, Levey AS, Uhlig K. Estimating equations for glomerular filtration rate in the era of creatinine standardization: a systematic review. Ann Intern Med. 2012;156(11):785-95. W-270, W$1-\mathrm{W}-8$.
5 Kidney Disease: Improving Global Outcomes (KDIGO) CKD Work Group. Comments on "KDIGO 2012 clinical practice guideline for the evaluation and management of chronic kidney disease". Kidney Int. 2013;84(3):6223.

6 Miller WG, Jones GRD. Estimated glomerular filtration rate; laboratory implementation and current global status. Adv Chronic Kidney Dis. 2018;25(1):7-13.

7 Levey AS, Coresh J, Tighiouart H, Greene T, Inker LA. Measured and estimated glomerular filtration rate: current status and future directions. Nat Rev Nephrol. 2020;16(1):51-64.
8 Pottel H, Bjork J, Courbebaisse M, Couzi L, Ebert N, Eriksen BO, et al. Development and validation of a modified full age spectrum creatinine-based equation to estimate glomerular filtration rate: a cross-sectional analysis of pooled data. Ann Intern Med. 2020;174(2): 183-91.

9 American Society of Nephrology. Available from: https://www.asn-online.org/g/blast/ files/NKF-ASN-eGFR-March2021.pdf. Accessed 2021 March 31 